\title{
Klimaanpassung in der Regionalplanung - Eine deutschlandweite Analyse zum Implementationsstand klimaanpassungsrelevanter Regionalplaninhalte
}

\author{
Hanna Christine Schmitt ${ }^{1}$
}

Eingegangen: 25. Mai 2015 / Angenommen: 31. Dezember 2015 / Online publiziert: 18. Januar 2016

(C) Springer-Verlag Berlin Heidelberg 2016

\begin{abstract}
Zusammenfassung Seit Veröffentlichung der „Deutschen Anpassungsstrategie an den Klimawandel“" herrscht wissenschaftlicher und planungspolitischer Konsens darüber, dass die Regionalplanung zur Implementation von Klimaanpassungsinhalten befähigt ist. Inwieweit die formelle Regionalplanung diese Befähigung gegenwärtig umsetzt, ist zentraler Untersuchungsgegenstand dieses Artikels. Die Analyse ist eine Vollerhebung für die Regionalplanungsgebiete Deutschlands. Deren Regionalpläne werden auf Vorhandensein und Verbindlichkeit von klimaanpassungsrelevanten Festlegungen untersucht. Das „Handlungskonzept der Raumordnung zu Vermeidungs-, Minderungsund Anpassungsstrategien im Hinblick auf die räumlichen Konsequenzen des Klimawandels" der Ministerkonferenz für Raumordnung und seine sieben klimaanpassungsbezogenen Handlungsfelder dienen als Analysegrundlage. Im Ergebnis wird der Stand der Implementation von klimaanpassungsrelevanten Regionalplaninhalten dargestellt sowohl für jedes Handlungsfeld als auch handlungsfeldübergreifend. Zudem werden die Ergebnisse reflektiert und weitere Anpassungspotenziale aufgezeigt.
\end{abstract}

Schlüsselwörter Regionalplanung · Klimawandel · Klimaanpassung · Adaption · Anpassungskapazität · Deutschland

Hanna Christine Schmitt, M.Sc.

hanna.schmitt@tu-dortmund.de

Fakultät Raumplanung, Institut für Raumplanung (IRPUD), TU Dortmund, August-Schmidt-Str. 10, 44227 Dortmund, Deutschland

\section{Climate Change Adaptation in Regional Planning-A Germany-Wide Analysis on the State of Implementation of Adaptation-Relevant Regional Plan Contents}

\begin{abstract}
Since the publication of the German Strategy for Adaptation to Climate Change there has been scientific and planning political consensus on the ability of regional planning to implement climate change adaptation contents. To which extent the formal regional spatial planning meets this ability, is the key issue of this article. The analysis is a census for the regional planning territories of Germany. Their regional plans are examined regarding the presence and bindingness of adaptation-relevant designations. The action plan of spatial planning on prevention, mitigation and adaptation strategies with regard to the spatial consequences of climate change of the Conference of Ministers on Spatial Planning and its seven adaptation-related fields of action serve as a basis for the analysis. As a result, the state of implementation of adaptation-relevant regional plan contents is presented - both for each field of action as well as comprehensively. Furthermore, the results are reflected and further adaptation potentials are presented.
\end{abstract}

Keywords Regional planning ' Climate change · Climate change adaptation - Adaptation .

Adaptive capacity $\cdot$ Germany

\section{Einleitung}

Der globale Klimawandel gehört zu den drängendsten Herausforderungen der planerischen Gegenwart. Denn der anthropogen genutzte Raum ist gegenüber den Auswirkungen des Klimawandels vulnerabel (verwundbar), 
woraus sich, unabhängig von ebenfalls wichtigen Klimaschutzbemühungen, eine Notwendigkeit zur Anpassung bestehender und zukünftiger räumlicher Strukturen und Raumnutzungen ergibt. Es gilt daher bereits gegenwärtig eine vorausschauende, klimaangepasste Planung zu etablieren, die sich der Persistenz ihrer Entscheidungen bewusst ist und über die Geltungsdauer aktueller Raumordnungspläne hinaus die verstärkten (und eventuell zusätzlichen) Auswirkungen des Klimawandels berücksichtigt (BMVBS/BBSR 2009, S. 5).

Der regionalen Raumordnungsebene (Regionalplanung) kommt in der „Herausforderung Klimaanpassung“ eine besondere Rolle zu. Als intermediäre Ebene zwischen rahmensetzender Bundes-/Landesplanung und umsetzender Bauleitplanung weist die Regionalplanung einen überfachlichen, überörtlichen und zugleich räumlich-konkreten Charakter auf, der sie im besonderen Maße dazu befähigt, vulnerabilitätsreduzierende Festlegungen $\mathrm{zu}$ treffen. Zudem ist die Regionalplanung seit Veröffentlichung der „Deutschen Anpassungsstrategie an den Klimawandel“" (DAS) (Bundesregierung 2008) und der Novellierung des Raumordnungsgesetzes (ROG) durch $\S 2$ Abs. 2 Nr. 6 ROG explizit dazu aufgefordert, einer klimaangepassten Raumentwicklung Rechnung zu tragen (Bundesregierung 2008, S. 7; BMVBS/BBSR 2009, S. 23; Birkmann/Fleischhauer 2013, S. 44; Frommer/Schlipf/Böhm et al. 2013, S. 128 f.). Eine Konkretisierung dieses Handlungsauftrages erfolgte auf der 36. Ministerkonferenz für Raumordnung (MKRO) durch den Beschluss des „Handlungskonzeptes der Raumordnung zu Vermeidungs-, Minderungs- und Anpassungsstrategien im Hinblick auf die räumlichen Konsequenzen des Klimawandels" ${ }^{\text {"1 }}$. Die MKRO definiert darin die folgenden sieben klimaanpassungsrelevanten Handlungsfelder: Vorbeugender Hochwasserschutz in Flussgebieten; Küstenschutz; Schutz der Berggebiete (insbesondere Alpenraum); Schutz vor Hitzefolgen in Siedlungsbereichen (bioklimatische Belastungsgebiete); Regionale Wasserknappheiten; Veränderungen im Tourismusverhalten; Verschiebung der Lebensräume von Tieren und Pflanzen (MKRO 2009; MKRO 2013).

Das Ziel dieses Artikels ist es, den Status quo der Implementation von klimaanpassungsrelevanten Inhalten in den Regionalplanungsregionen Deutschlands darzustellen. Die Analyse ist auf die Auswertung der rechtsverbindlichen Regionalpläne fokussiert, da diese als formelle Instrumente direkte Bindungswirkung entfalten und deutschlandweit flächendeckend vorliegen. Als ,grundsätzlich klimaanpassungsrelevant" werden die sieben oben genannten Handlungsfelder des MKRO-Handlungskonzeptes und ihre Handlungsschwerpunkte verstanden. Zur Operationalisierung des Implementationsstandes konkreter

\footnotetext{
${ }^{1}$ Nachfolgend als „MKRO-Handlungskonzept“ bezeichnet.
}

klimaanpassungsrelevanter Regionalplaninhalte werden aus den Handlungsschwerpunkten Festlegungen abgeleitet, auf deren Vorhandensein und Verbindlichkeit (Festlegung als Ziele der Raumordnung/Ausweisung von Vorranggebieten; Festlegung als Grundsätze der Raumordnung/Ausweisung von Vorbehaltsgebieten) alle Regionalpläne Deutschlands untersucht werden (siehe Kap. 2).

Die Analyseergebnisse werden zunächst handlungsfeldübergreifend ausgewertet und reflektiert, sodass ein Gesamtbild zum Implementationsstand klimaanpassungsrelevanter Regionalplaninhalte gezeichnet wird (siehe Kap. 3). Anschließend erfolgt eine handlungsfeldbezogene Bewertung und Reflexion der Analyseergebnisse. Diese wird in den gegenwärtigen Wissenskontext eingebettet, der im Wesentlichen durch die Forschungsstudien und Projektergebnisse der Bundesforschungsprogramme KLIMZUG (gefördert vom Bundesministerium für Bildung und Forschung (BMBF) von 2008 bis 2014) und KlimaMORO (gefördert von Bundesministerium für Verkehr, Bau und Stadtentwicklung (BMVBS) und Bundesinstitut für Bau-, Stadt- und Raumforschung (BBSR) von 2009 bis 2013) geprägt ist. Insbesondere die Ergebnisse der KlimaMORO-Vorstudie werden zur Kontextualisierung der Analyseergebnisse herangezogen, da diese den Stand der Raumordnungspraxis im Umgang mit Klimaschutz und Klimaanpassung vor Veröffentlichung des MKROHandlungskonzeptes widerspiegelt (siehe Kap. 4).

Der Artikel zielt auf die Identifizierung weiterer Anpassungspotenziale und soll zu einer zielgerichteten Umsetzung der Herausforderung Klimaanpassung auf wissenschaftlicher und auf praktischer Planungsebene beitragen.

Wenngleich in die Analyse des Implementationsstandes keine informellen Instrumente einbezogen werden, so sei an dieser Stelle dennoch auf ihre besondere Relevanz im Umgang mit der Herausforderung Klimaanpassung hingewiesen, denn diese können maßgeblich sowohl zur Vorbereitung als auch zur Umsetzung formeller Instrumente beitragen und somit ihre Wirksamkeit erhöhen (BMVBS/ BBSR 2009, S. 29 f.).

\section{Methodisches Vorgehen}

Das MKRO-Handlungskonzept definiert sieben Handlungsfelder zur Anpassung an den Klimawandel, die auf regionaler und lokaler Planungsebene umgesetzt werden sollen. Für eine deutschlandweite Analyse des Status quo der Regionalplanung in der Klimaanpassung sind insbesondere die formellen Regionalpläne als Analysebasis geeignet, da diese gemäß $\S 8$ Abs. 1 Nr. 2 ROG für die Teilräume der Länder, das heißt flächendeckend, aufzustellen sind. Darüber hinaus sind die Festlegungen der Regionalpläne für nachgeordnete Planungen bindend und auf der 
Tab. 1 Handlungsfelder und Handlungsschwerpunkte der Klimaanpassung (eigene Darstellung nach Bayrische Staatsregierung 2013, S. 36-38; MKRO 2013)

Handlungsfeld I - Vorbeugender Hochwasserschutz in Flussgebieten

\begin{tabular}{|c|c|}
\hline I.i & $\begin{array}{l}\text { Sicherung vorhandener Überschwemmungsbereiche als } \\
\text { Retentionsraum }\end{array}$ \\
\hline I.ii & $\begin{array}{l}\text { Rückgewinnung von Überschwemmungsbereichen als } \\
\text { Retentionsraum }\end{array}$ \\
\hline I.iii & Risikovorsorge in potenziellen Überflutungsbereichen \\
\hline I.iv & $\begin{array}{l}\text { Verbesserung des Wasserrückhaltes in der Fläche der Ein- } \\
\text { zugsgebiete der Flüsse }\end{array}$ \\
\hline I.v & $\begin{array}{l}\text { Sicherung potenzieller Standorte für } \\
\text { Hochwasserschutzmaßnahmen }\end{array}$ \\
\hline \multicolumn{2}{|c|}{ Handlungsfeld II - Küstenschutz } \\
\hline II.i & $\begin{array}{l}\text { Ergänzende Risikominimierung in sturmflutgeschützten } \\
\text { Küstengebieten }\end{array}$ \\
\hline II.ii & $\begin{array}{l}\text { Risikominimierung in nicht ausreichend sturmflut- } \\
\text { geschützten Küstengebieten }\end{array}$ \\
\hline II.iii & $\begin{array}{l}\text { Freihaltung von Pufferzonen an ungeschützten } \\
\text { Erosionsküsten }\end{array}$ \\
\hline II.iv & $\begin{array}{l}\text { Raumbedarf für Klei- und marine Sandentnahmestellen zu } \\
\text { Küstenschutzzwecken sichern }\end{array}$ \\
\hline II.v & $\begin{array}{l}\text { Freihaltung von Bereichen vor und hinter Küstenschutz- } \\
\text { anlagen von konkurrierenden Nutzungen }\end{array}$ \\
\hline
\end{tabular}

Handlungsfeld III - Schutz der Berggebiete (insbesondere

Alpenraum)

III.i $\quad$ Erhalt/Wiederherstellung der Schutzfunktionen des Bergwaldes

III.ii Schutz vor (Berg-)Naturgefahren

III.iii Sicherung und Weiterentwicklung der Berggebiete als Lebens-, Wirtschafts- und Tourismusraum

Handlungsfeld IV - Schutz vor Hitzefolgen in Siedlungsbereichen (bioklimatische Belastungsgebiete)

IV.i Schutz überörtlich bedeutsamer klimawirksamer Freiräume/ Ausgleichsflächen

IV.ii Räumliche Steuerung der Siedlungsflächen- und Infrastrukturentwicklung

Handlungsfeld V - Regionale Wasserknappheiten

V.i Sicherung von Wasserressourcen

V.ii Unterstützung des Erhalts bzw. der Verbesserung des Wasserhaushaltes der Böden

V.iii Vorausschauende Lenkung stark wasserverbrauchender Nutzungen

Handlungsfeld VI - Veränderungen im Tourismusverhalten

VI.i Festlegung neuer Tourismusschwerpunkte und Entwicklungsräume

VI.ii Standortsicherung für tourismusbezogene Infrastruktur Handlungsfeld VII - Verschiebung der Lebensräume von Tieren und Pflanzen

VII.i Sicherung eines überörtlichen, funktional zusammenhängenden Netzes ökologisch bedeutsamer Freiräume

VII.ii Minimierung weiterer Zerschneidungen

Ebene der Bauleitplanung weiter zu konkretisieren, sodass eine Umsetzung der Inhalte auf lokaler Ebene sichergestellt ist (ARL 2005, S. 969 f.).

Zur Analyse des Implementationsstandes klimaanpassungsrelevanter Regionalplaninhalte werden die sieben MKRO-Handlungsfelder zunächst in Handlungsschwerpunkten konkretisiert. Die Handlungsschwerpunkte stammen überwiegend aus dem MKRO-Handlungskonzept, sind für Handlungsfeld III jedoch aus dem Alpenplan generiert. Tabelle 1 beinhaltet die in der Analyse verwendeten Handlungsfelder und -schwerpunkte.

Für jeden dieser 22 klimaanpassungsrelevanten Handlungsschwerpunkte werden zur Systematisierung der Analyse Festlegungen definiert, deren Vorhandensein und Verbindlichkeit mittels einer inhaltlich-qualitativen Desktopanalyse untersucht wird. Die Bildung von Suchworten strukturiert den Analyseprozess. Ein Beispiel für die verwendeten klimaanpassungsrelevanten Festlegungen und Suchworte kann Tabelle 2 entnommen werden.

Mit diesem methodischen Vorgehen wurden sämtliche rechtskräftigen Regionalpläne der Bundesrepublik Deutschland analysiert und in einer Datenbank hinterlegt. ${ }^{2}$

Die Verbindlichkeit der klimaanpassungsrelevanten Festlegungen in den einzelnen Handlungsschwerpunkten bildet das Kernelement der Analyse und dient der Bewertung des Implementationsstandes und der Ermittlung des weiteren Anpassungspotenzials. Festlegungen, die als Ziele der Raumordnung (oder als Ausweisung von Vorranggebieten) erfolgen, sind endgültig abgewogen und entfalten daher eine Beachtungspflicht für nachgeordnete Planungen. Aufgrund der hohen Bindungswirkung werden Zielfestlegungen als 100-prozentige Implementation gewertet. Festlegungen, die als Grundsätze der Raumordnung (oder als Ausweisung von Vorbehaltsgebieten) erfolgen, sind im Einzelnen abwägungsrelevant, in nachgeordneten Planungen mit geringerer Bindungswirkung zu berücksichtigen und werden daher als 50-prozentige Implementation gewertet. Planungshinweise entfalten keine Bindungswirkung, sodass der Implementationsstand bei diesen mit null Prozent gewertet wird. Wenngleich die Klassifizierung der verschiedenen Festlegungsarten anhand ihrer Bindungswirkung eine Simplifizierung der tatsächlichen Wirksamkeit von Zielen, Grundsätzen und Planungshinweisen darstellt, ermöglicht dieses quantitative Vorgehen eine differenzierte und systematische Bewertung des Implementationsstandes.

Da nicht alle Handlungsfelder für sämtliche Planungsregionen relevant sind, wird in die Bewertung zudem der Charakter der Planungsregionen einbezogen. Während davon ausgegangen werden kann, dass die Handlungsfelder I sowie IV bis VII für jede Planungsregion Relevanz besitzen, werden außerdem Küstenregionen (Handlungsfeld II ist relevant) und Bergregionen (Handlungsfeld III ist relevant) abgegrenzt und davon die sonstigen Regionen (Handlungsfelder II und III sind nicht relevant) unterschieden. Küstenregionen sind als solche Regionen

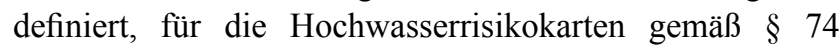

\footnotetext{
${ }^{2}$ Die Datenbank kann auf Nachfrage von der Autorin bezogen werden.
} 
Tab. 2 Klimaanpassungsrelevante Festlegungen und Suchworte für Handlungsschwerpunkt I.i

\begin{tabular}{lll}
\hline $\begin{array}{l}\text { Handlungsfelder und } \\
\text { Handlungsschwerpunkte }\end{array}$ & Klimaanpassungsrelevante Festlegungen & Suchworte \\
\hline I- Vorbeugender Hochwasserschutz in Flussgebieten & Hochwasserschutz \\
\hline I.i $\quad \begin{array}{l}\text { Sicherung vorhandener Über- } \\
\text { schwemmungsbereiche als }\end{array}$ & - Ausweisung von Vorranggebieten für die Überschwemmungsflächen & Hochwasser/ \\
Retentionsraum & eines HQ100, & Hochwasser* (für z. B. \\
& -Ausweisung von Vorbehaltsgebieten für die Überschwemmungsflächen & Hochwasserschutz) \\
& eines HQextrem & Überschwemmung \\
& zur vorsorglichen Sicherung von noch nicht wasserrechtlich festgesetzten & Retention \\
& Überschwemmungsflächen und zum Ausschluss entgegenstehender & HQ \\
& Nutzungen (MKRO 2013, S. 14; BMVBS/BBSR 2013, S. 70). &
\end{tabular}

Wasserhaushaltsgesetz (WHG) vorliegen. Von diesen gibt es insgesamt 23, die sich in den Bundesländern Mecklenburg-Vorpommern, Niedersachsen und Schleswig-Holstein befinden. ${ }^{3}$ Als Bergregionen werden allen voran die drei Alpenregionen Allgäu, Oberland und Südost-Oberbayern verstanden. Da die Handlungsschwerpunkte des Handlungsfeldes III Schutz der Berggebiete jedoch auch für die deutschen Mittelgebirgsregionen relevant sind, werden die drei Alpenregionen um sämtliche Planungsregionen mit Bergen ab 500 Höhenmetern (mangels einer allgemeingültigen Definition) ergänzt, sodass sich in der Summe 50 Regionen ergeben, für die Handlungsfeld III relevant ist. Da es keine Planungsregion gibt, die zugleich Küsten- als auch Bergregion ist, existieren 38 sonstige Regionen.

Unter Berücksichtigung des Charakters der Planungsregionen ergibt sich die Berechnung des handlungsfeldübergreifenden (Gesamt-) Implementationsstandes klimaanpassungsrelevanter Regionalplaninhalte auf Grundlage der Normalisierung der handlungsfeldbezogenen Bewertung. Eine Planungsregion besitzt demnach einen Implementationsstand von $100 \%$, wenn in allen Handlungsschwerpunkten der sieben MKRO-Handlungsfelder die klimaanpassungsrelevanten Festlegungen als Ziele der Raumordnung (Vorranggebiete) erfolgen. Die Normalisierung erlaubt den Vergleich von Planungsregionen unterschiedlichen Charakters und ermöglicht eine Auswertung nach Bundesländern. Ob die Zielfestlegung für die Handlungsschwerpunkte im Einzelfall jedoch angemessen ist, kann mit dieser quantitativen Analysemethode nicht bewertet werden und steht hinter dem Ziel einer deutschlandweiten Vollerhebung zurück.

Des Weiteren werden die Regionalpläne auf das Vorhandensein eines „Kapitels Klimaanpassung ${ }^{64}$ und auf die Verwendung der Begriffe der Handlungsfeld-Titel (z. B. „Hitzefolgen“) untersucht. Hierdurch sollen Erkenntnisse

\footnotetext{
${ }^{3}$ Die Stadtstaaten Bremen und Hamburg besitzen ebenfalls Hochwasserrisikokarten, entfallen in der Analyse jedoch aufgrund ihrer regionalplanerischen Besonderheiten (siehe Kap. 3).

${ }^{4}$ Kapiteltitel: „Klimaanpassung“; „Klimaschutz und Klimaanpassung"; „Anpassung an den Klimawandel“; „Mitigation und Adaption“; o. Ä.
}

darüber generiert werden, ob die Klimaanpassung gegenwärtig zur Begründung eines regionalplanerischen Handlungserfordernisses herangezogen wird.

\section{Handlungsfeldübergreifende Ergebnisse und kritische Reflexion}

Die Analyse bezieht ausschließlich Regionalpläne ein, die am Stichtag 30.06.2014 rechtsverbindlich vorlagen. Nicht berücksichtigt sind daher beispielsweise zu diesem Zeitpunkt in Aufstellung befindliche Pläne und nicht integrierte Regionalplanänderungen. Insgesamt umfasst die Analyse 111 Regionalpläne aus zwölf Bundesländern. Nicht berücksichtigt sind Sonderformen der Regionalplanung, die in den Landesraumordnungsgesetzen verankert sind (in Abb. 1 in Grau dargestellt). Dies betrifft die Stadtstaaten Berlin, Bremen (einschließlich Bremerhaven), Hamburg, das Saarland, die kreisfreien Städte Niedersachsens und die Städteregion Ruhr. ${ }^{5}$ Berücksichtigt sind hingegen Regionalpläne, die aufgrund des Versäumnisses der Pflicht zur Prüfung des Änderungs- oder Fortschreibungsbedarfs oder der Aktualisierung der Pläne gemäß § 5 (7) des niedersächsischen Landesraumordnungsgesetzes (NROG) am Stichtag unwirksam waren (Landkreise Aurich und Oldenburg). Im Sinne der Analyse erfüllen diese keines der Handlungsfelder und erzielen daher einen Implementationsstand von null Prozent (in Abb. 1 in Hellgrün dargestellt). Die Planungsregionen Brandenburgs erzielen in der Analyse

\footnotetext{
${ }^{5}$ Gemäß $§ 8$ (1) Satz 3 ROG besteht die Möglichkeit, dass in den Stadtstaaten ein Flächennutzungsplan (FNP) nach $\S 5$ BauGB die Funktionen eines Landesentwicklungsplanes (LEP) übernimmt, sodass die Stadtstaaten (und auch das Saarland) von der Verpflichtung zur Aufstellung von Regionalplänen befreit sind. Ebenfalls keinen Regionalplan besitzen die sechs kreisfreien Städte Niedersachsens, deren Befreiung von der Planaufstellungspflicht in $\S 5$ Abs. 2 NROG geregelt ist. Die Planungsregion Städteregion Ruhr besitzt gemäß § 8 Abs. 4 ROG statt eines Regionalplans einen Regionalen Flächennutzungsplan (RFNP). Dieser übernimmt so, im Einklang mit ROG und Baugesetzbuch (BauGB) geregelt, zugleich die Funktionen von Regional- und Flächennutzungsplan.
} 


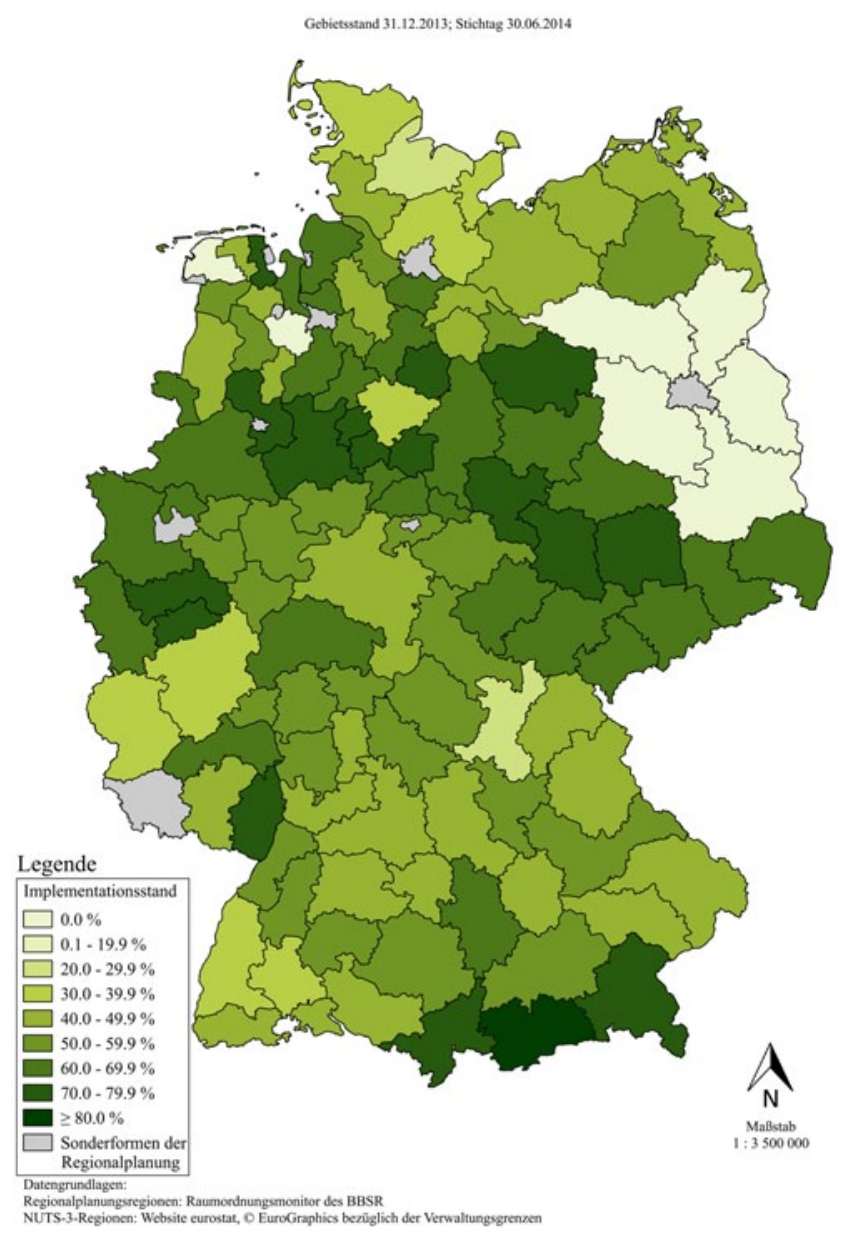

Abb. 1 Handlungsfeldübergreifender Implementationsstand klimaanpassungsrelevanter Regionalplaninhalte

ebenfalls null Prozent, da diese am Stichtag ausschließlich über sachliche Teilpläne verfügten.

Abbildung 1 veranschaulicht eine starke Heterogenität in den Implementationsständen klimaanpassungsrelevanter Regionalplaninhalte der deutschen Planungsregionen. Abgesehen von den Landkreisen Aurich und Oldenburg sowie den Planungsregionen Brandenburgs erzielen alle Planungsregionen einen handlungsfeldübergreifenden Implementationsstand von über $20 \%$. Gegenwärtig erzielt jedoch nur die Planungsregion Oberland einen Implementationsstand von über $80 \%$. Der bundesdurchschnittliche Status quo der Implementation von klimaanpassungsrelevanten Regionalplaninhalten lag am Stichtag 30.06.2014 bei $52,71 \%$.

Werden die handlungsfeldübergreifenden Ergebnisse auf Bundesländerebene aggregiert, zeigt sich, dass in fünf Bundesländern ein unter- und in sieben Bundesländern ein überdurchschnittlicher Implementationsstand erzielt wird (siehe Tab. 3). Es ist auffällig, dass die drei Bundesländer mit den höchsten Implementationsständen (Sachsen-Anhalt, Sachsen, Nordrhein-Westfalen) überwiegend aus Planungs-
Tab. 3 Implementationsstand klimaanpassungsrelevanter Regionalplaninhalte nach Bundesländern

\begin{tabular}{ll}
\hline Bundesland & $\begin{array}{l}\text { Durchschnittlich erzielter } \\
\text { Implementationsstand (\%) }\end{array}$ \\
\hline Brandenburg & 0,0 \\
Schleswig-Holstein & 37,9 \\
Baden-Württemberg & 47,3 \\
Mecklenburg-Vorpommern & 47,6 \\
Rheinland-Pfalz & 51,2 \\
Bayern & 53,1 \\
Hessen & 53,9 \\
Niedersachsen & 56,6 \\
Thüringen & 58,8 \\
Nordrhein-Westfalen & 61,5 \\
Sachsen & 66,2 \\
Sachsen-Anhalt & 72,9 \\
\hline
\end{tabular}

regionen sonstigen Charakters bestehen und somit wenige bis keine Berg- oder Küstenregionen aufweisen.

Der geringere handlungsfeldübergreifende Implementationsstand von Bundesländern mit Küsten- oder Bergregionen ist im Wesentlichen darauf zurückzuführen, dass in den Handlungsfeldern II - Küstenschutz und III - Schutz der Berggebiete bisher lediglich in geringem Maße klimaanpassungsrelevante Festlegungen getroffen werden. In den Handlungsfeldern IV - Schutz vor Hitzefolgen in Siedlungsbereichen und VII - Verschiebung der Lebensräume von Tieren und Pflanzen bestehen hingegen in fast allen Regionen relevante Festlegungen, sodass der Implementationsstand in diesen Handlungsfeldern bei jeweils ca. $90 \%$ liegt. In den verbleibenden Handlungsfeldern I - Vorbeugender Hochwasserschutz in Flussgebieten, V - Regionale Wasserknappheiten und VI - Veränderungen im Tourismusverhalten beträgt der Implementationsstand durchschnittlich zwischen 43 und $53 \%$ (siehe Tab. 4). Eine vertiefende Darstellung, Bewertung und Reflexion der handlungsfeldbezogenen Analyseergebnisse ist Kapitel 4 zu entnehmen.

Die Analyse ergibt des Weiteren, dass zwischen dem Jahr des Inkrafttretens der Regionalpläne und ihrem Implementationsstand eine positive Korrelation besteht. Demnach weisen tendenziell jüngere Regionalpläne einen höheren Implementationsstand auf als ältere. Da allerdings Regionalpläne, die zwischen den Jahren 2000 und 2008 in Kraft traten, im Durchschnitt einen höheren Implementationsstand $(59,23 \%)$ aufweisen als Regionalpläne, die ab 2009 in Kraft traten (56,54\%), ist die positive Korrelation eher schwach ausgeprägt. ${ }^{6}$ Verglichen mit dem durchschnittlichen Implementationsstand der Regionalpläne, die vor dem Jahr 2000 in Kraft traten (49,47\%), zeigt sich insgesamt eine Zunahme klimaanpassungsrelevanter Inhalte in den jüngeren Regionalplänen von bis zu zehn Prozentpunkten.

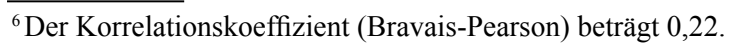


Tab. 4 Implementationsstand klimaanpassungsrelevanter Regionalplaninhalte nach Handlungsfeldern

\begin{tabular}{|c|c|c|c|c|c|c|c|}
\hline & $\begin{array}{l}\text { HF I Vor- } \\
\text { beugender } \\
\text { Hochwasser- } \\
\text { schutz in } \\
\text { Flussgebieten }\end{array}$ & $\begin{array}{l}\text { HF II } \\
\text { Küstenschutz }\end{array}$ & $\begin{array}{l}\text { HF III } \\
\text { Schutz der } \\
\text { Berggebiete } \\
\text { (insbesondere } \\
\text { Alpenraum) }\end{array}$ & $\begin{array}{l}\text { HF IV Schutz vor } \\
\text { Hitzefolgen in } \\
\text { Siedlungsbereichen }\end{array}$ & $\begin{array}{l}\text { HF V Regionale } \\
\text { Wasserknappheiten }\end{array}$ & $\begin{array}{l}\text { HF VI Ver- } \\
\text { änderungen im } \\
\text { Tourismusverhalten }\end{array}$ & $\begin{array}{l}\text { HF VII Ver- } \\
\text { schiebung } \\
\text { der Lebens- } \\
\text { räume von } \\
\text { Tieren und } \\
\text { Pflanzen } \\
\end{array}$ \\
\hline $\begin{array}{l}\text { Prozentualer } \\
\text { Implementations- } \\
\text { stand }(\%)\end{array}$ & 43,6 & 18,2 & 16,0 & 88,5 & 44,3 & 52,5 & 89,5 \\
\hline
\end{tabular}

Ein Grund dafür, dass die seit 2009 in Kraft getretenen Regionalpläne durchschnittlich einen etwas niedrigeren Implementationsstand klimaanpassungsrelevanter Inhalte aufweisen, kann in der Fokussierung der Regionalplanung auf die räumliche Steuerung der Windenergienutzung gesehen werden, die mit Inkrafttreten der Novellierung des Erneuerbare-Energien-Gesetzes (EEG) im Jahre 2009 erforderlich wurde (BBSR 2015, S. 6). Die vorrangige Auseinandersetzung mit der Windenergienutzung zeigt sich beispielsweise in den brandenburgischen Regionalplanungsregionen, die zum Stichtag der Analyse die Aufstellung integrierter Regionalpläne ,zugunsten von Teilplänen [...] zurückgestellt“" hatten (Landesplanungsabteilung Berlin-Brandenburg 2015).

Als weiterer Grund ist anzunehmen, dass aufgrund der zeitaufwendigen Verfahren zur Änderung und Neuaufstellung von Regionalplänen bisher die wenigsten der seit 2009 in Kraft getretenen Regionalpläne Inhalte des Aktionsplans Anpassung (APA) (Bundesregierung 2011) oder der Forschungsprogramme KLIMZUG und KlimaMORO (abgeschlossen seit 2013/2014) beinhalten. Es bleibt demnach abzuwarten, ob die derzeit in Aufstellung oder Änderung befindlichen Regionalpläne entsprechend höhere Implementationsstände erzielen.

Zuletzt ist bei der Bewertung der Analyseergebnisse zu berücksichtigen, dass aufgrund der rein quantitativen Analysemethode keine Rückschlüsse darauf gezogen werden können, ob die jüngeren Regionalpläne eventuell trotz eines scheinbar geringeren Implementationsstandes eine qualitativ zielführende Ausweisung klimaanpassungsrelevanter Inhalte vornehmen, indem beispielsweise strengere Grenzwerte (z. B. $\mathrm{HQ}_{\text {extrem }}$ statt $\mathrm{HQ}_{100}$ ) Verwendung finden. Regionalspezifische Analysen, die auf dieser Analyse zum Vorhandensein und der Verbindlichkeit klimaanpassungsrelevanter Inhalte aufbauen und sowohl Ausgestaltung als auch Angemessenheit der Implementation untersuchen, könnten diese Erkenntnislücke schließen.

Bezüglich der Fragestellung, ob die Klimaanpassung in den Regionalplänen im Allgemeinen zur Begründung eines regionalplanerischen Handlungserfordernisses herangezogen wird, ergibt die Analyse ein deutliches Ergebnis. Gegenwärtig verfügen vier der 111 Regionalpläne über ein separates „Kapitel Klimaanpassung“. Da alle vier Regionalpläne nach 2009 in Kraft traten, kann von einer gewissen Steigerung des Bewusstseins einzelner Regionalplanungsregionen für die Herausforderung Klimaanpassung gesprochen werden. Die Analyse zur Begriffsverwendung der Handlungsfeld-Titel in den Planwerken zeigt, dass lediglich der Begriff „Hochwasserschutz“ (aufgrund von historischer Betroffenheit und fachlich-politischer Thematisierung) flächendeckend Verwendung findet. Begriffe wie „Schutz der Berggebiete“, „Hitzefolgen“, „Wasserknappheiten“ und auch „Lebensräume von Tieren und Pflanzen“ finden hingegen in sehr wenigen Regionalplänen Erwähnung.

Aus den Analyseergebnissen lässt sich die Erkenntnis ableiten, dass die Klimaanpassung bisher nur in geringem Maße Einzug in die Regionalplanungspraxis gehalten hat, denn sie wird in den seltensten Fällen zur Begründung eines regionalplanerischen Handlungserfordernisses angeführt. Bisher werden klimaanpassungsrelevante Festlegungen überwiegend indirekt getroffen, und zwar hauptsächlich in Handlungsfeldern, welche die raumstrukturellen Kernaufgaben der Regionalplanung nach $\S 8$ Abs. 5 ROG behandeln (Siedlungs-, Freiraum- und Infrastrukturentwicklung). Aus diesem Grund erzielen auch Regionalpläne, die vor dem Jahr 2000 in Kraft traten, einen durchschnittlichen Implementationsstand von knapp 50\%.

Es zeigt sich demnach deutlich, dass im Umgang mit der Herausforderung Klimaanpassung in den formellen Regionalplänen Deutschlands weitere Anpassungspotenziale bestehen. Diese Anpassungspotenziale beschränken sich nicht allein auf eine langfristige Erhöhung des Implementationsstandes handlungsfeldbezogener Regionalplaninhalte. Vielmehr bestehen diese in einer Stärkung der Relevanz des Themas Klimaanpassung in der Regionalplanung, sodass dieses als eigenständiges Handlungserfordernis wahrgenommen und umgesetzt wird.

\section{Handlungsfeldbezogene Ergebnisse und kritische Reflexion}

\subsection{Handlungsfeld I - Vorbeugender Hochwasserschutz in Flussgebieten}

In nahezu allen Regionalplänen Deutschlands werden klimaanpassungsrelevante Festlegungen in Handlungs- 


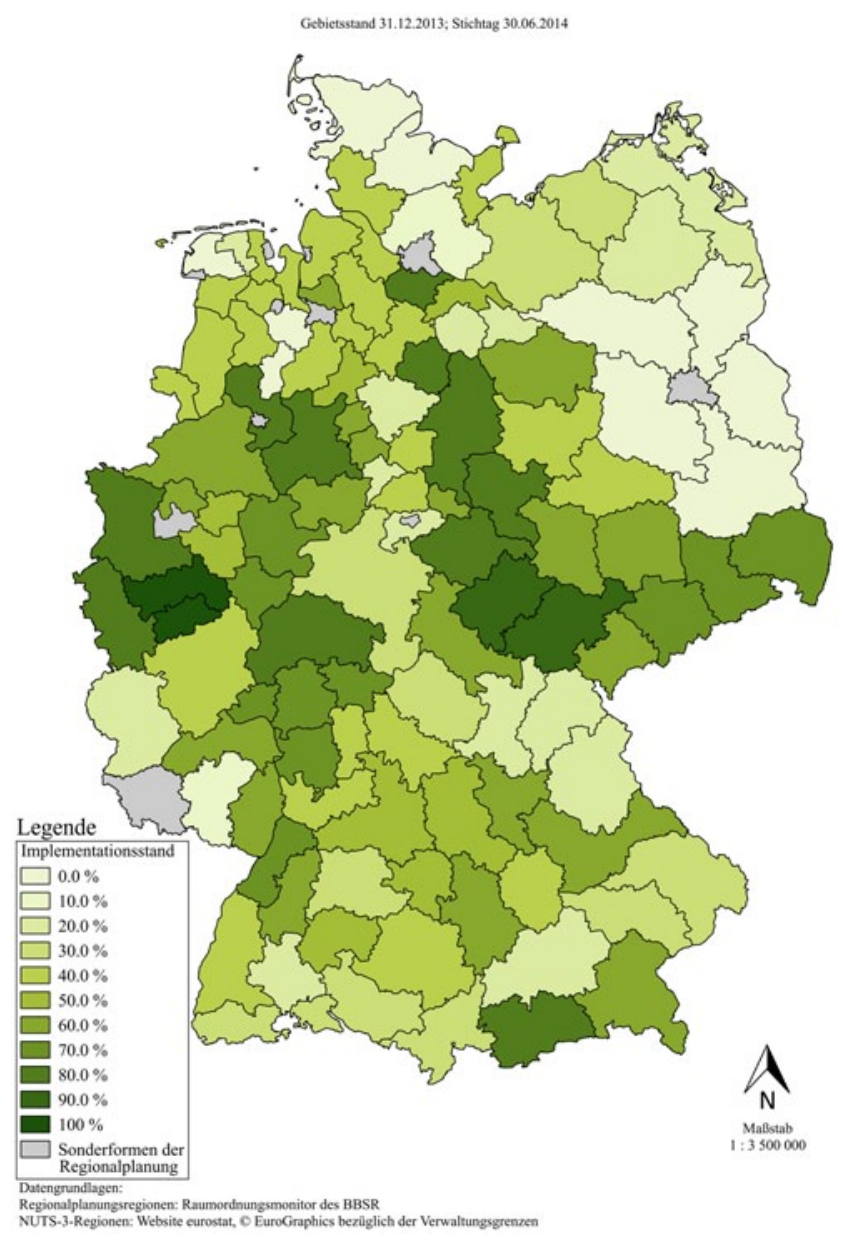

Abb. 2 Implementationsstand in Handlungsfeld I - Vorbeugender Hochwasserschutz in Flussgebieten

feld I - Vorbeugender Hochwasserschutz in Flussgebieten getroffen. Dieser Umstand ist unter anderem auf die von der MKRO veröffentlichten „Handlungsempfehlungen zum vorbeugenden Hochwasserschutz" (2000) sowie die Novellierung des WHG zurückzuführen (MKRO 2013, S. 14) (Abb. 2).

Bezüglich der Ausweisung von Vorrang- und Vorbehaltsgebieten Hochwasserschutz ist ein Vergleich der Analyseergebnisse mit den Ergebnissen der KlimaMORO-Vorstudie möglich. Während im Analysezeitraum 2008/2009 in 56 Regionalplänen Vorranggebiete und in 22 Regionalplänen Vorbehaltsgebiete zum Hochwasserschutz ausgewiesen waren (BMVBS 2010, S. 75), verfügen im Jahr 201490 Regionalpläne entweder über Zielfestlegungen zum Hochwasserschutz oder weisen entsprechende Vorranggebiete aus.

Dennoch zeigt der bundesdurchschnittliche Implementationsstand von $43,6 \%$, dass in Handlungsfeld I weitere Anpassungspotenziale existieren. Besonderer Anpassungsbedarf besteht in Handlungsschwerpunkt I.iii - Risikovorsorge in potenziellen Überflutungsbereichen, da dieser

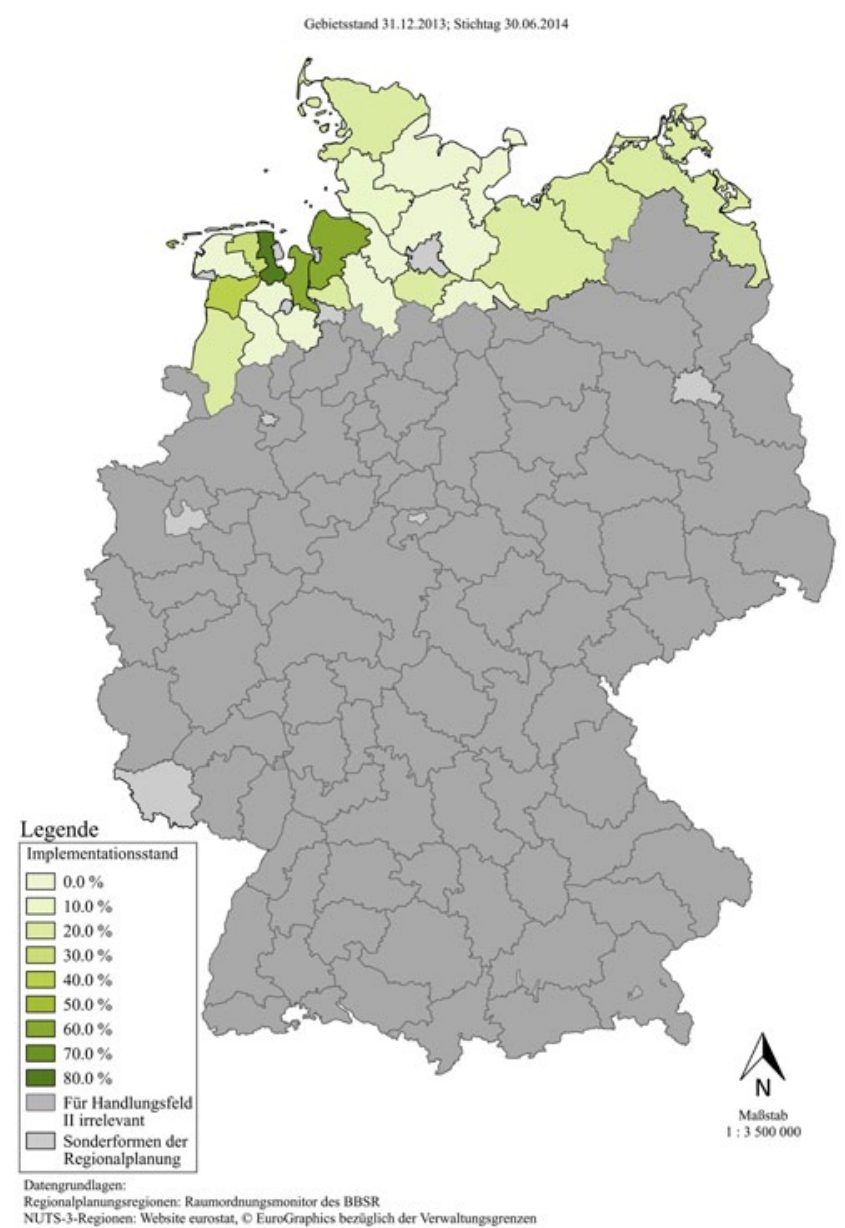

Abb. 3 Implementationsstand in Handlungsfeld II - Küstenschutz

bisher von lediglich ca. einem Fünftel der Planungsregionen verbindlich festgelegt wird. Ein Grund hierfür ist die verhältnismäßig junge Auseinandersetzung mit dem Einfluss des Klimawandels auf den vorbeugenden Hochwasserschutz in Flussgebieten, die maßgeblich durch die EU-Hochwasserrisikomanagement-Richtlinie (HWRM-RL 2007) initiiert wurde. Bei der Umsetzung der „Risikovorsorge in potenziellen Überflutungsbereichen" können informelle Verfahren und Instrumente zu einem besseren Risikoverständnis und einer verstärkten Risikokommunikation im Rahmen raumordnerischer Zusammenarbeit beitragen (MKRO 2013, S. 18, 22).

Des Weiteren zeigt die Analyse am Beispiel der Bundesländer Schleswig-Holstein und Mecklenburg-Vorpommern, dass Festlegungen in Handlungsfeld I vorrangig in formellen Fachplanungsinstrumenten wie beispielsweise dem „Generalplan Binnenhochwasserschutz SchleswigHolstein" getroffen werden. Sofern die in den Fachplanungsdokumenten enthaltenen Festlegungen (noch) nicht in die Regionalpläne integriert wurden, können diese in der Analyse des Implementationsstandes nicht erfasst werden. 


\subsection{Handlungsfeld II - Küstenschutz}

In Handlungsfeld II - Küstenschutz wird gegenwärtig durchschnittlich ein Fünftel aller klimaanpassungsrelevanten Regionalplaninhalte als Ziel oder Grundsatz festgelegt. Es ergibt sich im Mittel aller Küstenregionen ein prozentualer Implementationsstand von 18,2\% (Abb. 3).

Gegenwärtig werden in Handlungsfeld II in zehn von 23 Küstenregionen keine Festlegungen getroffen. Ein wesentlicher Grund hierfür ist, dass die in der Analyse verwendeten Gebietsdefinitionen von Küstenregionen auf den Hochwasserrisikokarten gemäß HWRM-RL basieren. Im Gegensatz zu früheren Definitionen, wie beispielsweise in der KlimaMORO-Vorstudie verwendet (BMBVS 2010, S. 75), definiert die HWRM-RL auch Regionalplanungsregionen ohne unmittelbaren Kontakt zur Küstenlinie als potenziell sturmflutgefährdet. Da die Regionalpläne der Küstenregionen jedoch alle bereits vor Inkrafttreten der HWRM-RL verbindlich wurden, berücksichtigen sie diese neue Definition bisher nicht. Zudem ist Handlungsschwerpunkt II.iii - Freihalten von Pufferzonen an ungeschützten Erosionsküsten nicht für alle Küstenregionen relevant, sodass es im Einzelnen zu einer leichten negativen Verzerrung der Ergebnisse führen kann.

Wie in Handlungsfeld I decken auch in Handlungsfeld II teilweise fachplanerische Instrumente die klimaanpassungsrelevanten Inhalte der MKRO-Handlungsschwerpunkte ab. Im „Generalplan Küstenschutz des Landes SchleswigHolstein" gelten beispielsweise die darin enthaltenen Entwicklungsziele zugleich als Ziele der Raumordnung ${ }^{7}$ (Innenministerium des Landes Schleswig-Holstein 2010, S. 122). Bezüglich einer Integration dieser Ziele in die Regionalpläne besteht weiteres Anpassungspotenzial.

Ein weiteres Anpassungspotenzial ist bezüglich des Handlungsschwerpunktes II.ii - Risikominimierung in nicht ausreichend sturmflutgeschützten Küstengebieten festzustellen, der bisher von lediglich einer Küstenregion thematisiert wird. Wie bereits bei der Hochwasserrisikovorsorge kann die Regionalplanung auch hier über informelle Verfahren und Instrumente $\mathrm{zu}$ einem besseren Problemverständnis von Risiko beitragen und somit eine wichtige Rolle in der Risikokommunikation und Risikominimierung einnehmen (MKRO 2013, S. 18, 22).

\footnotetext{
${ }^{7}$ Darin heißt es konkret: „Der Küstenschutz in Schleswig-Holstein ist auf Grundlage des ,Generalplans Küstenschutz: Integriertes Küstenschutzmanagement in Schleswig-Holstein $(\mathrm{GPK})^{6}$ in der jeweils gültigen Fassung zu gewährleisten. Die dort enthaltenen Entwicklungsziele sind zugleich Ziele der Raumordnung. In den Regionalplänen sind raumordnerische Ziele für die einzelnen Küstenschutzmaßnahmen und Deichlinien zu konkretisieren“"(Innenministerium des Landes Schleswig-Holstein 2010, S. 122).
}

\subsection{Handlungsfeld III - Schutz der Berggebiete}

Die drei Handlungsschwerpunkte des Handlungsfeldes III - Schutz der Berggebiete sind in Ermangelung von Inhalten im MKRO-Handlungskonzept aus dem Alpenplan Bayern abgeleitet. Handlungsfeld III ist mit durchschnittlich $16,0 \%$ das Handlungsfeld mit dem geringsten Implementationsstand.

Abbildung 4 verdeutlicht die starken Unterschiede, die zwischen den Alpen- und den sonstigen Bergregionen bestehen. Diese resultieren insbesondere aus der weit gefassten Definition von Berggebieten, die nichtsdestotrotz für die Analyse angemessen ist, da eine Betroffenheit durch den Klimawandel auch für die Mittelgebirgsregionen zu erwarten ist (BMVBS/BBSR 2013, S. 117; MKRO 2013, S. 23).

Die meisten klimaanpassungsrelevanten Festlegungen finden sich zu Handlungsschwerpunkt III.i - Erhalt/Wiederherstellung der Schutzfunktionen des Bergwaldes, was überwiegend auf $\S 12$ des Bundeswaldgesetzes (BWaldG) zurückzuführen ist. Darin heißt es: „Die Erklärung zu Schutzwald kommt insbesondere in Betracht zum Schutz

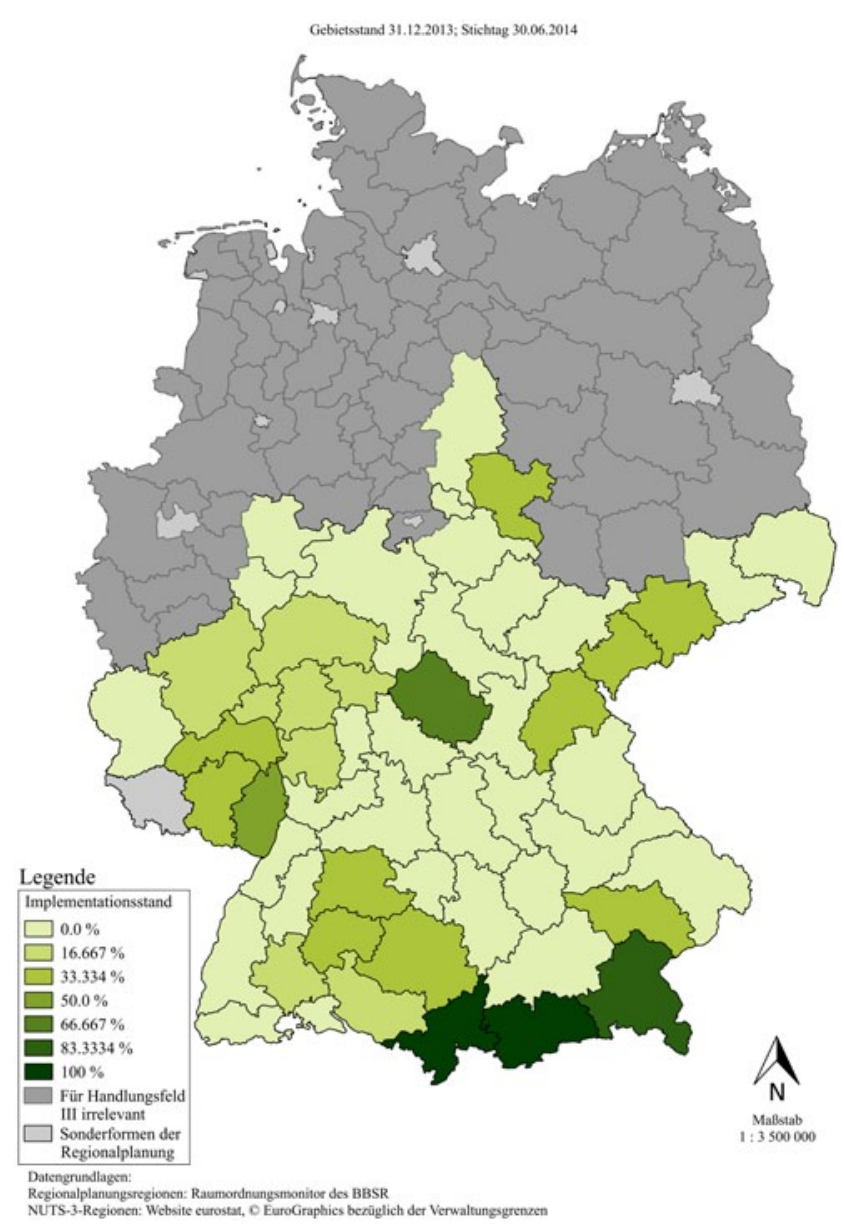

Abb. 4 Implementationsstand in Handlungsfeld III - Schutz der Berggebiete 


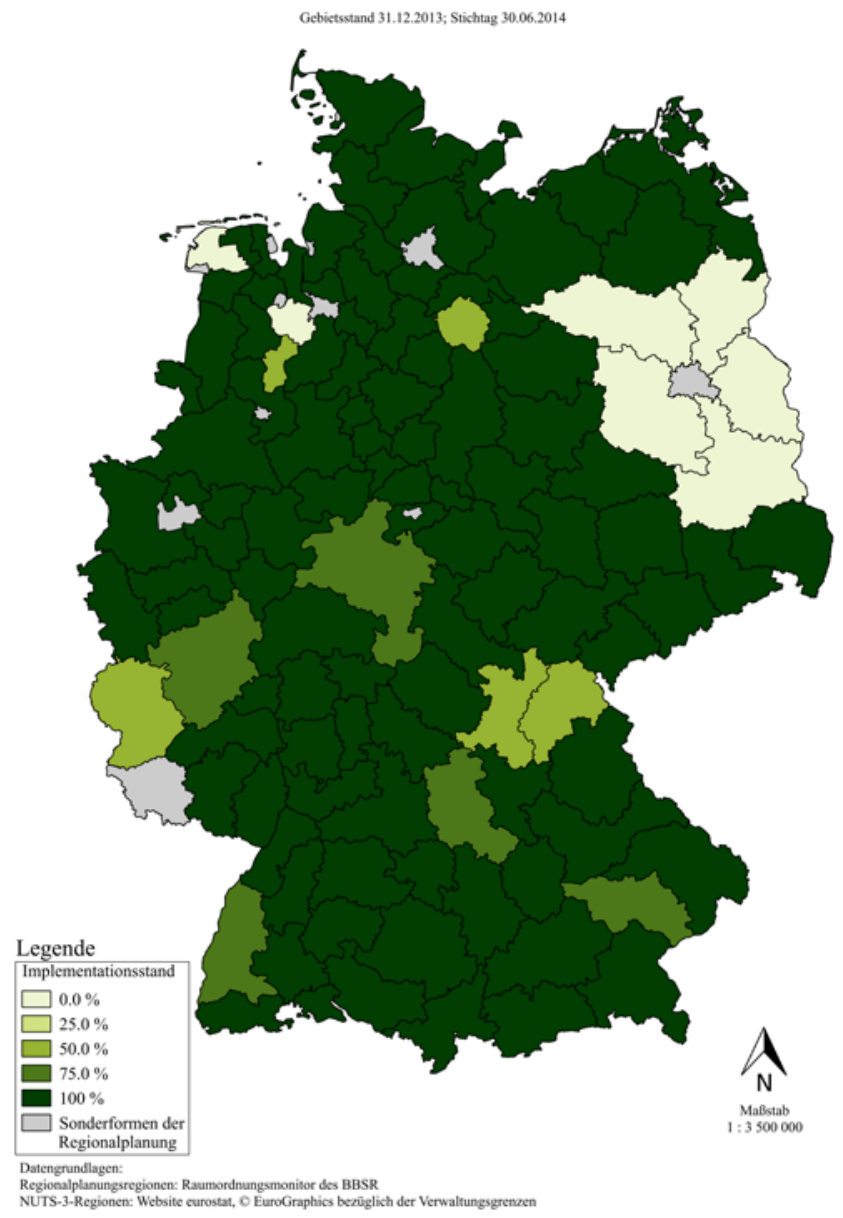

Abb. 5 Implementationsstand in Handlungsfeld IV - Schutz vor Hitzefolgen in Siedlungsbereichen

gegen schädliche Umwelteinwirkungen im Sinne des Bundes-Immissionsschutzgesetzes [...], Erosion durch Wasser und Wind, Austrocknung, schädliches Abfließen von Niederschlagswasser und Lawinen“ (§ 12, Satz 2 BWaldG). Darüber hinaus ist ein deutliches Anpassungspotenzial zum Umgang mit geogenen Naturgefahren festzustellen.

Vor dem Hintergrund einer $\mathrm{zu}$ erwartenden starken klimawandelbedingten Betroffenheit in Berggebieten ${ }^{8}$ ist das Anpassungspotenzial und der Anpassungsbedarf in Handlungsfeld III besonders dringend. Die gezielte Verwendung von informellen Instrumenten in der Regionalplanung kann dabei einen wichtigen Beitrag leisten. Durch Regionalmanagementinitiativen und Risiko-GovernanceProzesse lässt sich beispielsweise zu einer Erhöhung des Risikobewusstseins, einer besseren Risikokommunikation

${ }^{8}$ Überdurchschnittliche Erwärmung (Zunahme Jahresmittel- und Winterhalbjahrestemperatur sowie Zunahme der heißen Tage über $30^{\circ} \mathrm{C}$ ), Reduzierung der Gletscher, Aufwärtsverschiebung der Vegetationszonen (Gefährdung der alpinen Flora und Fauna), Zunahme von Starkniederschlägen und in Folge dessen Hochwassergefahr, Zunahme von Steinschlag, Muren und Lawinen (BMVBS/ BBSR 2013, S. 117; MKRO 2013, S. 23). und $\mathrm{zu}$ einer Umsetzung von Risikokonzepten beitragen (MKRO 2013, S. 24).

\subsection{Handlungsfeld IV - Schutz vor Hitzefolgen in Siedlungsbereichen}

Die zwei Handlungsschwerpunkte des Handlungsfeldes IV - Schutz vor Hitzefolgen in Siedlungsbereichen sind vornehmlich als Zielfestlegungen in den Regionalplänen verankert, sodass sich im Bundesdurchschnitt ein deutlich überdurchschnittlicher Implementationsstand von $88,5 \%$ ergibt (Abb. 5).

Während im Analysezeitraum der KlimaMOROVorstudie (2008/2009) 79 Regionalpläne eine Ausweisung von Regionalen Grünzügen vornahmen (BMVBS 2010, S. 79), beinhalten im Jahr 2014 alle rechtskräftigen Regionalpläne Ausweisungen zum „Schutz überörtlich bedeutsamer klimawirksamer Freiräume/Ausgleichsflächen“, die überwiegend ebenfalls durch Regionale Grünzüge erfolgen. Dieser Implementationsfortschritt ist positiv zu bewerten. Kritisch zu reflektieren ist jedoch, dass aufgrund der quantitativen Analysemethode nicht nachvollzogen werden kann, ob die Ausweisungen klimaanpassungsbezogen oder im Kontext der Freiraumsicherung erfolgen. Da in lediglich einem Regionalplan der Begriff des HandlungsfeldTitels „Hitzefolgen“ verwendet wird, ist davon auszugehen, dass die Ausweisung der Regionalen Grünzüge bisher eher freiraumbezogen erfolgt und der Klimaanpassung somit indirekt dienlich ist. Es bestehen daher Anpassungspotenziale sowohl in der Ausweitung von Festlegungen mit eindeutigem Klimaanpassungsbezug als auch in der Stärkung einer klimaanpassungsbezogenen Perspektive auf bestehende Flächenkategorien der Freiraumsicherung.

\subsection{Handlungsfeld V - Regionale Wasserknappheiten}

Die drei Handlungsschwerpunkte des Handlungsfeldes V Regionale Wasserknappheiten sind gegenwärtig mit einem durchschnittlichen Implementationsstand von $44,33 \%$ festgelegt (Abb. 6).

Bezüglich der Ausweisung von Vorranggebieten zum Grundwasserschutz lässt sich gegenüber dem Analysezeitraum der KlimaMORO-Vorstudie ein Implementationsfortschritt feststellen. Während im Analysezeitraum 2008/2009 72 Regionalpläne Vorranggebiete zum Grundwasserschutz auswiesen (BMVBS 2010, S. 79), werden im Jahr 2014 in 87 Regionalplänen Vorranggebiete ausgewiesen oder Zielfestlegungen zum Grundwasserschutz getroffen. In 14 Regionalplanungsregionen erfolgt bisher jedoch keine Ausweisung von Grundwasserschutzgebieten, was insbesondere vor dem Hintergrund klimatischer Veränderungen, die eine Veränderung der Qualität und Verfügbarkeit von Grund- und 


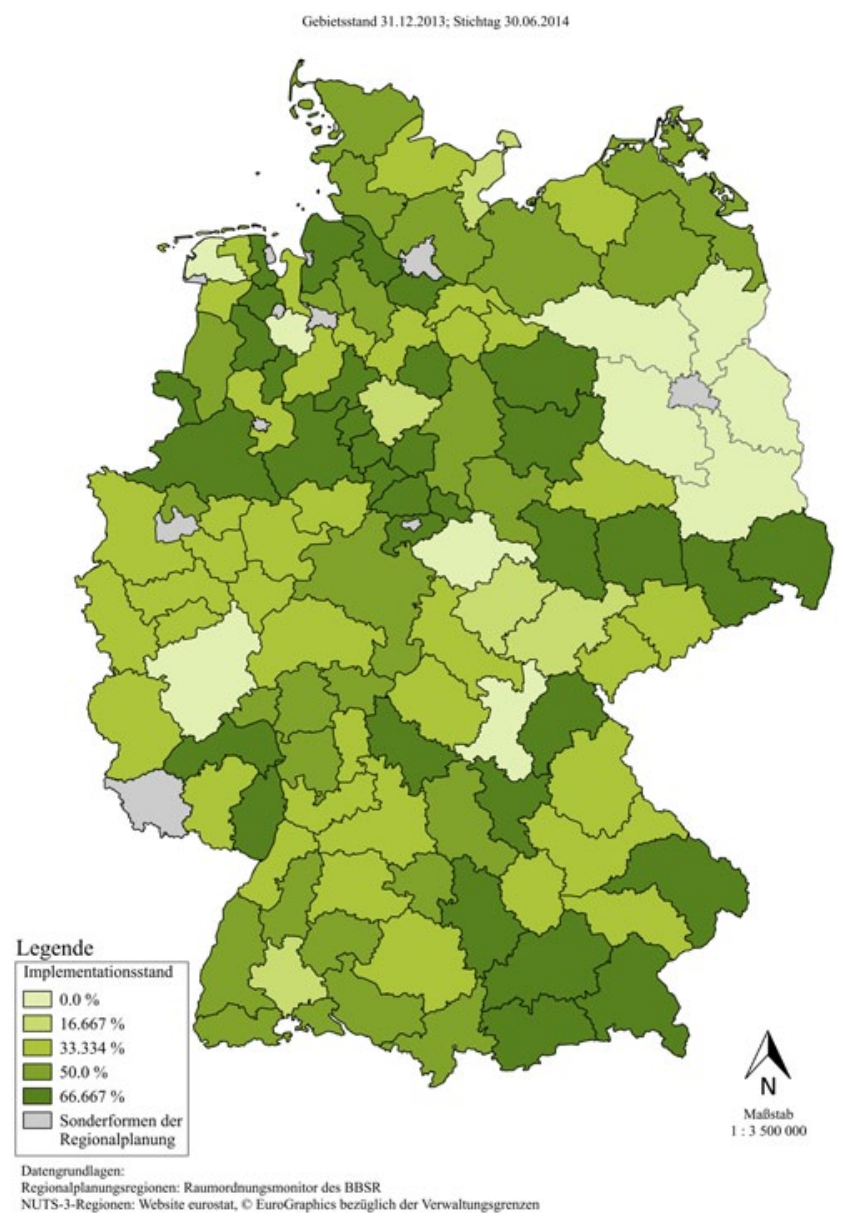

Abb. 6 Implementationsstand in Handlungsfeld V - Regionale Wasserknappheiten

Oberflächenwasser erwarten lassen, kritisch zu beurteilen ist (MKRO 2013, S. 28).

In Handlungsschwerpunkt V.ii - Unterstützung des Erhalts bzw. der Verbesserung des Wasserhaushaltes der Böden lässt sich ebenfalls weiteres Anpassungspotenzial identifizieren, da in knapp $40 \%$ der Regionen hierzu keine Festlegungen getroffen wurden. Die Regionalplanung kann diesbezüglich beispielsweise im Rahmen der informellen Regionalentwicklung $\mathrm{zu}$ einer besseren Implementation beitragen, indem sie die Moderation im Prozess der Identifizierung und Sicherung besonders sensibler Bereiche der Grundwassereinzugsgebiete übernimmt (MKRO 2013, S. 29).

Auffällig ist in Handlungsfeld V des Weiteren, dass gegenwärtig keine Regionalplanungsregion Festlegungen in Handlungsschwerpunkt V.iii - Vorausschauende Lenkung stark wasserverbrauchender Nutzungen trifft. Eine mögliche Begründung dafür ist, dass es an einer geeigneten Flächenkategorie zur „Sicherung von Bereichen für stark wasserverbrauchende Nutzungen" beziehungsweise an Festlegungen von Ausschlussbereichen für diese mangelt. Eine andere Begründung könnte sein, dass die Analyse in

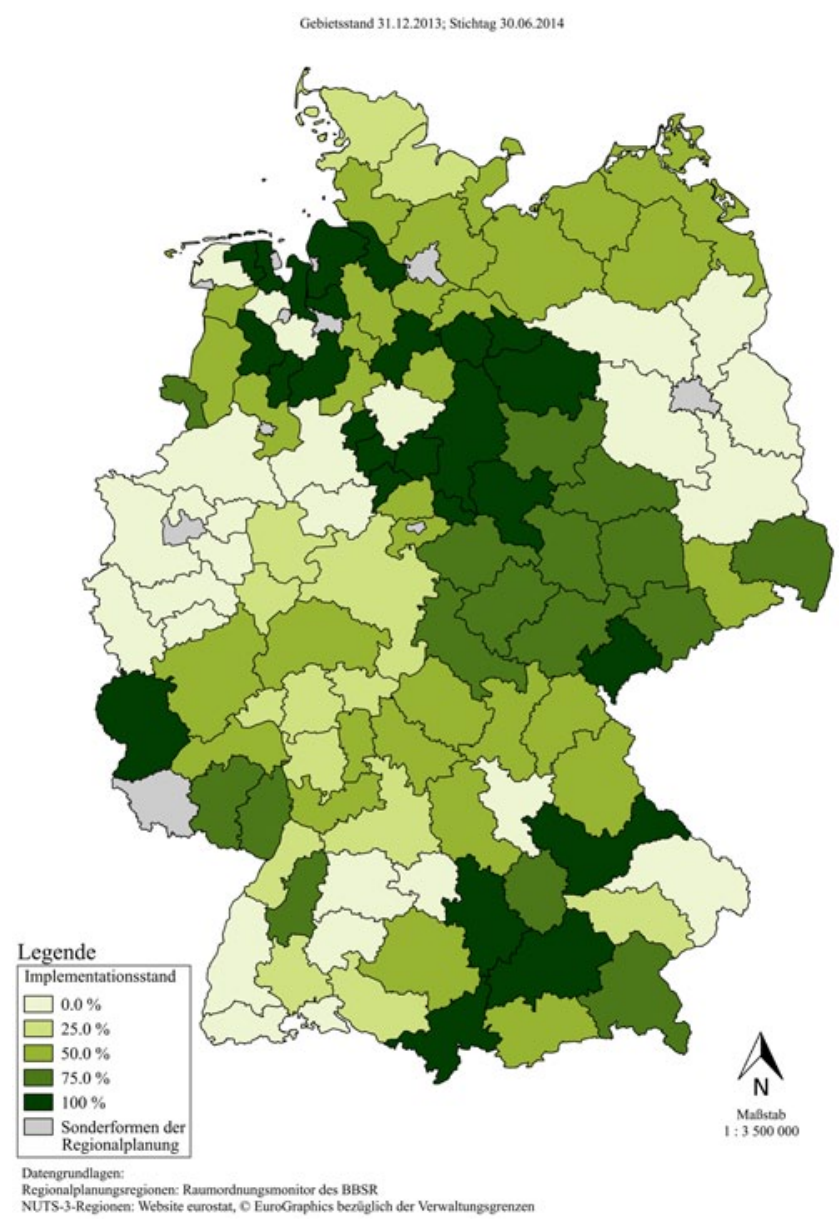

Abb. 7 Implementationsstand in Handlungsfeld VI - Veränderungen im Tourismusverhalten

diesem Handlungsschwerpunkt womöglich zu eng gefasst wurde, sodass Festlegungen, die im weiteren Sinne unter Handlungsschwerpunkt V.iii fallen, nicht abgedeckt werden konnten. ${ }^{9}$

\subsection{Handlungsfeld VI - Veränderungen im Tourismusverhalten}

Im Sinne der Sicherung eines sanften Tourismus besteht eine flächendeckende Relevanz zur Koordination der Tourismusentwicklung aufgrund des sich verändernden Tourismusverhaltens (BfN 2011). Die Regionalplanung beeinflusst diese bisher eher über informelle Instrumente und Strategien (MKRO 2013, S. 31). Dennoch beträgt der durchschnittliche Implementationsstand klimaanpassungsrelevanter Regionalplaninhalte in Handlungsfeld VI - Veränderungen im Tourismusverhalten 52,5\%.

\footnotetext{
${ }^{9}$ Beispielsweise finden sich sowohl in den Landesentwicklungsplänen und -programmen als auch in einigen Regionalplänen Festlegungen zur „Einschränkung des Wasserverbrauchs der gewerblichen Wirtschaft".
} 
In Handlungsfeld VI ist auffällig, dass die Implementationsstände der Regionen zwischen den Bundesländern deutlich variieren. Dieser Umstand weist darauf hin, dass die regionale Implementation dieses Handlungsfeldes stark von der Landesraumordnungsebene und den Festlegungen in den Landesraumordnungsplänen und -programmen abhängig ist. Auch in der KlimaMOROVorstudie wird darauf verwiesen, dass in Regionalplänen sehr wenig bis keine tourismusbezogene Steuerung stattfindet (BMVBS 2010, S. 79).

Abbildung 7 zeigt auch, dass ausgerechnet in vielen Küsten- und Bergregionen, die sowohl gegenwärtig als auch zukünftig als Haupttourismusziele in Deutschland gelten (Bundesregierung 2008, S. 44), kaum oder gar keine Zielfestlegungen erfolgen. Lediglich in Niedersachsen, dem Bundesland, in dem die Regionalplanung auf der Ebene der Landkreise und kreisfreien Städte stattfindet und damit deutlich kleinräumiger als in anderen Ländern ausfällt, sind die zwei Handlungsschwerpunkte überwiegend als Ziele festgelegt. Die Begründung hierfür findet sich im Landesraumordnungsprogramm (LROP) Niedersachsen in den Grundsätzen 3.2.3-1 und 3.2.3-5, welche die Sicherung des Tourismus in der Natur und an den Küsten lenken (Niedersächsisches Ministerium für Ernährung, Landwirtschaft, Verbraucherschutz und Landesentwicklung 2008, S. 26 f.).

\subsection{Handlungsfeld VII - Verschiebung der Lebensräume von Tieren und Pflanzen}

In Handlungsfeld VII - Verschiebung der Lebensräume von Tieren und Pflanzen erzielen die Regionalpläne einen durchschnittlichen Implementationsstand von 89,5\%. Dieser hohe Implementationsstand ist Resultat zahlreicher nationaler und internationaler Gesetze und Strategiepapiere ${ }^{10}$ zum Arten- und Naturschutz (Abb. 8).

$\mathrm{Da}$ Wanderungsbewegungen von Arten kaum prognostizierbar sind, haben Festlegungen zum Umgang mit der „Verschiebung der Lebensräume von Tieren und Pflanzen“ in der Regel den Charakter von „No-Regret Strategien" (planerischen Strategien, die so ausgelegt werden, dass sie auch unter veränderten Rahmenbedingungen voraussichtlich keine negativen Auswirkungen haben werden). Die Festlegungen der Handlungsschwerpunkte VII.i - Sicherung von unzerschnittenen Freiräumen und VII.ii - Minimierung weiterer Zerschneidungen erfolgen demnach überwiegend indirekt, beispielsweise über die Ausweisung von Regionalen Grünzügen, die gegenwärtig jedoch in erster Linie der Freiraumsicherung dienen.

\footnotetext{
${ }^{10}$ Zum Beispiel: Grundsätze 2 und 6 des $\S 2$ Abs. 2 ROG und $\S 3$ des Bundesnaturschutzgesetzes (BNatSchG). Natura 2000-Netz in der Europäischen Union, bestehend aus Schutzgebieten der VogelschutzRichtlinie und der Flora-Fauna-Habitat-Richtlinie.
}

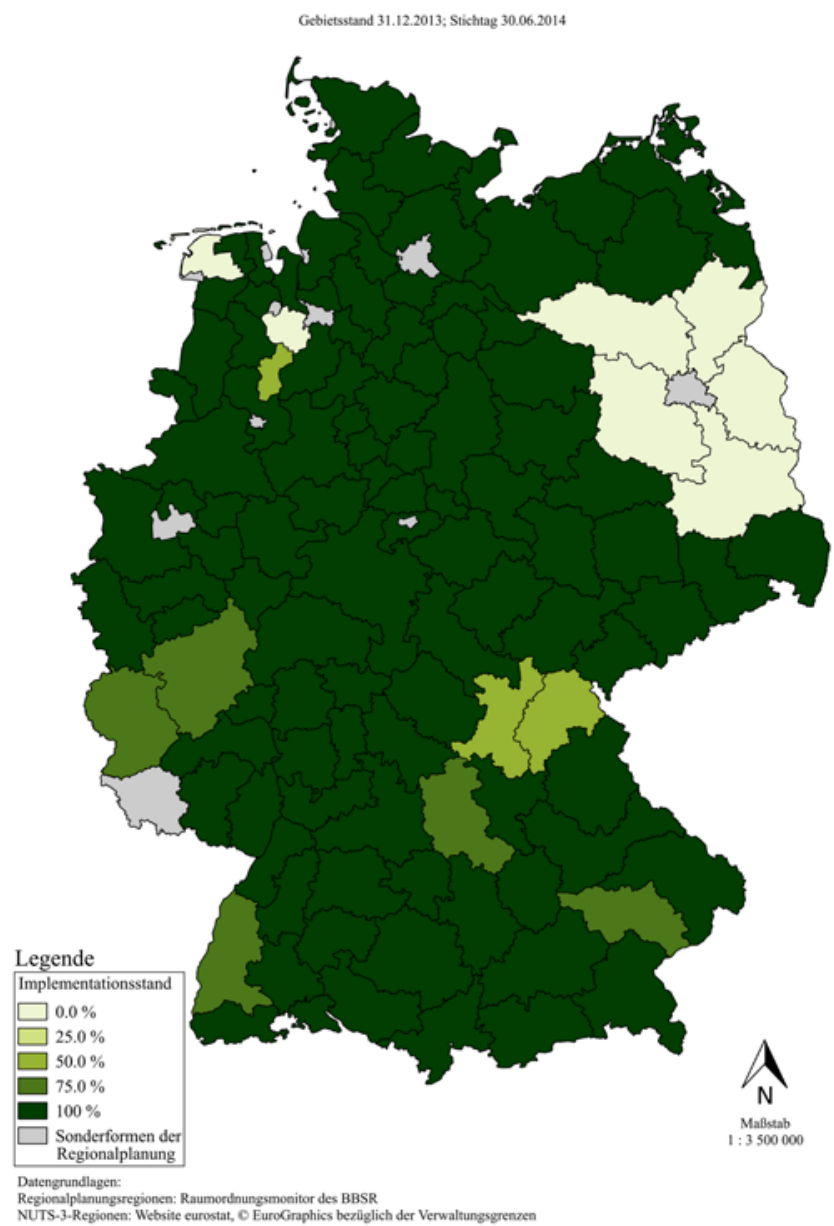

Abb. 8 Implementationsstand in Handlungsfeld VII - Verschiebung der Lebensräume von Tieren und Pflanzen

Wie auch in Handlungsfeld IV - Schutz vor Hitzefolgen in Siedlungsbereichen besteht daher ein Anpassungspotenzial in der Stärkung einer klimaanpassungsbezogenen Perspektive auf die Freiraumsicherung, sodass Festlegungen zukünftig mit dem Ziel des Erhalts und des Ausbaus der Lebensräume von Tieren und Pflanzen erfolgen können.

\section{Fazit}

Durch die Analyse aller rechtskräftigen Regionalpläne Deutschlands auf Vorhandensein und Verbindlichkeit klimaanpassungsrelevanter Festlegungen wurde der gegenwärtige Implementationsstand der Klimaanpassung in diesen abgebildet und es wurden weitere Anpassungspotenziale aufgezeigt. Die Darstellung des aktuellen Status quo leistet damit einen wichtigen Beitrag zur Operationalisierung der bisherigen Entwicklung des regionalplanerischen Umgangs mit der Herausforderung Klimaanpassung. Es lassen sich sowohl Rückschlüsse auf den derzeitigen Implementationsfortschritt ziehen als auch zukünftige Entwicklungen in der 
Gegenüberstellung mit dem derzeitigen Implementationsstand evaluieren. Auf regionaler Ebene können die Analyseergebnisse zudem als Grundlage für die Identifizierung und Priorisierung weiterer Maßnahmen zur Klimaanpassung dienen.

Aufgrund des rein quantitativen Charakters der Analyse, welcher Voraussetzung zur Systematisierung der Vollerhebung war, lassen sich im Einzelfall keine Rückschlüsse auf die Angemessenheit der Ausgestaltung der Festlegungen ziehen. Auch führt der alleinige Fokus auf die Regionalpläne dazu, dass in Fällen mangelnder Integration gleichwertig verbindliche Inhalte anderer formeller Instrumente nicht berücksichtig werden konnten. Zudem zeigte sich in mehreren Handlungsfeldern die Wichtigkeit informeller Instrumente, die zur Vorbereitung und Umsetzung der Regionalpläne und für deren Flexibilisierung große Relevanz besitzen, in der Analyse jedoch ebenfalls nicht berücksichtigt werden konnten.

Bezogen auf die einzelnen Handlungsfelder bestätigte sich die Aussage aus der KlimaMORO-Vorstudie, dass die Regionalplanung in vielen Handlungsfeldern zwar über einen „profunden Kenntnisstand“ (BMVBS 2010, S. 79) verfügt, dass der Handlungsauftrag zur Klimaanpassung jedoch nicht deutlich genug definiert ist. Dies zeigt sich beispielsweise in Handlungsfeld III - Schutz der Berggebiete (insbesondere Alpenraum), in dem es bisher an einem Handlungsauftrag zur Klimaanpassung in den Mittelgebirgsregionen mangelt. Ebenso zeigt sich in Handlungsfeld V - Regionale Wasserknappheiten, dass es an einer geeigneten (klimaanpassungsbezogenen) Flächenkategorie zur vorausschauenden Lenkung stark wasserverbrauchender Ressourcen fehlt.

Insgesamt zeigte sich, dass die Klimaanpassung gegenwärtig in den seltensten Fällen zur Begründung eines regionalplanerischen Handlungserfordernisses angeführt wird und dass der Umgang mit dem Thema Klimaanpassung in den Regionalplänen bisher eher indirekt erfolgt. Es wurde des Weiteren deutlich, dass der Implementationsstand klimaanpassungsbezogener Regionalplaninhalte in Handlungsfeldern, die gemäß $\S 8$ Abs. 5 ROG unter die raumstrukturellen Kernaufgaben der Regionalplanung fallen, deutlich höher als in anderen Handlungsfeldern ist. Ein allgemeines Anpassungspotenzial besteht demnach neben der handlungsfeldbezogenen Erhöhung des Implementationsstandes in der Stärkung der Relevanz der Herausforderung Klimaanpassung, sodass diese als eigenständiges Handlungserfordernis wahrgenommen und umgesetzt wird.

\section{Rechtsquellen}

BauGB - Baugesetzbuch in der Fassung der Bekanntmachung vom 23. September 2004 (BGBl. I S. 2414), geändert durch Artikel 6 des Gesetzes vom 20. Oktober 2015 (BGBl. I S. 1722).

BNatSchG - Gesetz über Naturschutz und Landschaftspflege (Bundesnaturschutzgesetz) in der Fassung der Bekanntmachung vom 29. Juli 2009 (BGBl. I S. 2542), zuletzt geändert durch Artikel 2 Absatz 124 des Gesetzes vom 7. August 2013 (BGBl. I S. 3154).

BWaldG - Gesetz zur Erhaltung des Waldes und zur Förderung der Forstwirtschaft (Bundeswaldgesetz) in der Fassung der Bekanntmachung vom 2. Mai 1975 (BGBl. I S. 1037), zuletzt geändert durch das Gesetz vom 31. Juli 2010 (BGBl. I S. 1050).

EEG - Erneuerbare-Energien-Gesetz in der Fassung der Bekanntmachung vom 25. Oktober 2008 (BGBl. I S. 2074), zuletzt geändert durch Artikel 1 des Gesetzes vom 12. April 2011 (BGBl. I S. 619).

Flora-Fauna-Habitat-Richtlinie - Richtlinie 92/43/EWG des Rates vom 21. Mai 1992 zur Erhaltung der natürlichen Lebensräume sowie der wildlebenden Tiere und Pflanzen.

HWRM-RL - Richtlinie 2007/60/EG des Europäischen Parlaments und des Rates vom 23. Oktober 2007 über die Bewertung und das Management von Hochwasserrisiken.

NROG - Niedersächsisches Raumordnungsgesetz in der Fassung der Bekanntmachung vom 18. Juli 2012 (Nds. GVB1. S. 252 - VORIS 23100 -), zuletzt geändert durch Artikel 1 des Gesetzes vom 25. Juni 2014 (Nds. GVBl. S. 168).

ROG - Raumordnungsgesetz in der Fassung der Bekanntmachung vom 22. Dezember 2008 (BGBI. I S. 2986), zuletzt geändert durch Artikel 9 des Gesetzes vom 31. Juli 2009 (BGBI. I S. 2585).

Vogelschutzrichtlinie - Richtlinie 2009/147/EG des Europäischen Parlaments und des Rates vom 30. November 2009 über die Erhaltung der wildlebenden Vogelarten.

WHG - Wasserhaushaltsgesetz in der Fassung der Bekanntmachung vom 31. Juli 2009 (BGBI. I S. 2585), zuletzt geändert durch Artikel 4 Absatz 76 des Gesetzes vom 7. August 2013 (BGBI. I S. 3154).

\section{Literatur}

ARL - Akademie für Raumforschung und Landesplanung (Hrsg.) (2005): Handwörterbuch der Raumordnung. Hannover.

Bayrische Staatsregierung (2013): Landesentwicklungsprogramm Bayern. München.

BfN - Bundesamt für Naturschutz (2011): Sanfter Tourismus. http:// www.bfn.de/0323_iyesanft.html (19.05.2015). 
Birkmann, J.; Fleischhauer, M. (2013): Vulnerabilität von Raumnutzungen, Raumfunktionen und Raumstrukturen. In: Birkmann, J.; Vollmer, M.; Schanze, J. (Hrsg.): Raumentwicklung im Klimawandel - Herausforderungen für die räumliche Planung. Hannover, 44-68. = Forschungsberichte der ARL 2.

BBSR - Bundesinstitut für Bau-, Stadt- und Raumforschung (Hrsg.) (2015): Steuerung der Windenergie durch die Regionalplanung - gestern, heute, morgen. Bonn. = BBSR-Analysen KOMPAKT 09/2015.

BMVBS - Bundesministerium für Verkehr, Bau und Stadtentwicklung (Hrsg.) (2010): Klimawandel als Handlungsfeld der Raumordnung: Ergebnisse der Vorstudie zu den Modellvorhaben „Raumentwicklungsstrategien zum Klimawandel“. Bonn.

BMVBS - Bundesministerium für Verkehr, Bau und Stadtentwicklung; BBSR - Bundesinstitut für Bau-, Stadt- und Raumforschung (Hrsg.) (2009): Entwurf eines regionalen Handlungs- und Aktionsrahmens Klimaanpassung („Blaupause“) - Ein Zwischenergebnis der Vorstudie für Modellvorhaben zu Raumentwicklungsstrategien zum Klimawandel. http://www.klimamoro.de/fileadmin/ Dateien/Ver\%C3\%B6ffentlichungen/Externe_Publikationen/ BBSR-Online-Publikation_17-09_Entwurf_eines_regionalen_ Handlungs-_und_Aktionsrahmens_Klimaanpassung_Blaupause. pdf. (26.11.2015). = BBSR-Online-Publikation 17/2009.

BMVBS - Bundesministerium für Verkehr, Bau und Stadtentwicklung; BBSR - Bundesinstitut für Bau-, Stadt- und Raumforschung (Hrsg.) (2013): Methodenhandbuch zur regionalen Klimafolgenbewertung in der räumlichen Planung - Systematisierung der Grundlagen regionalplanerischer Klimafolgenbewertung. Berlin/ Bonn.

Bundesregierung (2008): Deutsche Anpassungsstrategie an den Klimawandel - vom Bundeskabinett am 17. Dezember 2008 beschlossen. http://www.bmub.bund.de/fileadmin/bmu-import/files/ pdfs/allgemein/application/pdf/das_gesamt_bf.pdf. (19.05.2015).

Bundesregierung (2011): Aktionsplan Anpassung der Deutschen Anpassungsstrategie an den Klimawandel - vom Bundeskabinett am 31. August 2011 beschlossen. http://www.bmub.bund.de/ fileadmin/bmu-import/files/pdfs/allgemein/application/pdf/ aktionsplan_anpassung_klimawandel_bf.pdf. (22.05.2015).
Frommer, B.; Schlipf, S.; Böhm, H. R.; Janssen, G.; Sommerfeldt, P. (2013): Die Rolle der räumlichen Planung bei der Anpassung an die Folgen des Klimawandels. In: Birkmann, J.; Vollmer, M.; Schanze, J. (Hrsg.): Raumentwicklung im Klimawandel - Herausforderungen für die räumliche Planung. Hannover, 120-148. = Forschungsberichte der ARL 2.

Innenministerium des Landes Schleswig-Holstein (Hrsg.) (2010): Landesentwicklungsplan Schleswig-Holstein 2010. Kiel.

Landesplanungsabteilung Berlin-Brandenburg (2015): Regionalplanung. http://gl.berlin-brandenburg.de/regionalplanung/index. html. (13.11.2015).

MKRO - Ministerkonferenz für Raumordnung (2009): Bericht des Hauptausschusses der Ministerkonferenz für Raumordnung (MKRO). Handlungskonzept der Raumordnung zu Vermeidungs-, Minderungs- und Anpassungsstrategien im Hinblick auf die räumlichen Konsequenzen des Klimawandels. http://www.bmvi. de/SharedDocs/DE/Anlage/StadtUndLand/LaendlicherRaum/ bericht-zum-beschluss-raumordnung-und-klimawandel.pdf? blob=publicationFile (19.05.2015).

MKRO - Ministerkonferenz für Raumordnung (2013): Umlaufbeschluss Raumordnung und Klimawandel. Handlungskonzept der Raumordnung zu Vermeidungs-, Minderungs- und Anpassungsstrategien im Hinblick auf die räumlichen Konsequenzen des Klimawandels vom 23.01.2013. http://www.bmvi.de/ SharedDocs/DE/Anlage/StadtUndLand/LaendlicherRaum/ mkro-handlungskonzept-klima.pdf? blob=publicationFile (19.05.2015).

Niedersächsisches Ministerium für Ernährung, Landwirtschaft, Verbraucherschutz und Landesentwicklung (Hrsg.) (2008): LandesRaumordnungsprogramm Niedersachsen in der Fassung vom 08. Mai 2008. Hannover. 